\title{
Task order choices in cognitive and perceptual-motor tasks: The cognitive-load-reduction (CLEAR) hypothesis
}

\author{
Rachel L. VonderHaar ${ }^{1}$ • Dawn M. McBride ${ }^{1}$ - David A. Rosenbaum ${ }^{2}$
}

Published online: 9 May 2019

(C) The Psychonomic Society, Inc. 2019

\begin{abstract}
A core question in the study of the dynamics of cognition is how tasks are ordered. Given two tasks, neither of which is prerequisite for the other and neither of which brings a clearly greater reward, which task will be done first? Few studies have addressed this question, though recent work has suggested one possible answer, which we here call the cognitive-load-reduction (CLEAR) hypothesis. According to the CLEAR hypothesis, there is a strong drive to reduce cognitive load (to "clear one's mind"). Given two tasks, one of which is more cognitively demanding than the other, the more cognitively demanding task will tend to be done first. We tested this prediction using a novel method inviting participants to freely choose when to perform each of $c=5,10$, or 15 items per category in item-generation tasks relative to $b=10$ box-moving tasks. The box-moving tasks were cognitively undemanding relative to the item generation tasks, whose cognitive difficulty presumably grew with $c$. A full half of our $n=122$ participants chose to complete all of the $c$ tasks before performing any of the $b$ tasks, and most other participants chose to complete a majority of the $c$ tasks before any of the $b$ tasks. This result is consistent with the CLEAR hypothesis. Speed on the box-moving task decreased the later the category-generation task was completed, supporting another CLEAR prediction. The general method used here provides direction for future work on task order choices in cognitive and perceptual-motor tasks.
\end{abstract}

Keywords Cognitive control $\cdot$ Precrastination $\cdot$ Prospective memory

How do individuals choose the order of the tasks they carry out? When one task is prerequisite to others, that task must come first, and when a task brings much greater reward than others, that task will probably come first as well. But when neither of these conditions holds and the order of tasks is flexible, it is up to the actor to decide for himself or herself what to do when.

Few studies have addressed the question of how task ordering occurs in these circumstances, which is interesting because such studies could potentially reveal the criteria or constraints that surround the scheduling of daily activities. However, a recent set of studies (Blinch \& DeWinne, 2019; Fournier et al., 2019; Fournier, Stubblefield, Dyre, \&

Dawn M. McBride

dmcbride@ilstu.edu

1 Department of Psychology, Illinois State University, Campus Box 4620, Normal, IL 61790-4620, USA

2 University of California, Riverside, Riverside, CA, USA
Rosenbaum, 2018; Rosenbaum, Gong, \& Potts, 2014, see also Rosenbaum et al., 2019) suggested one possible basis for task ordering. The studies suggested, albeit indirectly, that decisions about task order may be driven by the desire to reduce cognitive effort. The basis for that suggestion will be explained in the brief research review to follow, but it is plausible because it is taxing to hold intentions in working memory. The burdensome nature of maintaining intentions in working memory has been shown in studies of prospective memory ( i.e., having to remember to do things in the future; e.g., Einstein \& McDaniel, 2005; Einstein, McDaniel, Manzi, Cochran, \& Baker, 2000; McDaniel, Einstein, Stout, \& Morgan, 2003). Insofar as the maintenance of intentions in working memory is taxing, if there is a way to get rid of that burden, that is what will occur. This idea also relates to work on other phenomena, such as reducing cognitive load in decision-making (e.g., Kool \& Botvinick, 2014; Kool, McGuire, Rosen, \& Botvinick, 2010).

Based on these findings, we now suggest the cognitiveload-reduction (CLEAR) hypothesis. The core claim of the CLEAR hypothesis is that individuals will "clear their minds" 
to the extent they can, at least in the midst of simple task choices. The prediction from the CLEAR hypothesis is that when given tasks to be done, tasks that are more cognitively demanding will be done sooner than tasks that are less cognitively demanding. To the best of our knowledge, the CLEAR hypothesis (or an analogous hypothesis not so named) has not been introduced before.

In the remainder of this article, we review the previous studies that have indirectly suggested the CLEAR hypothesis. Then we report an experiment designed to test the hypothesis directly. In the last part of the article, having presented data that support the CLEAR hypothesis, we turn to remaining issues.

\section{Previous studies suggesting the preference for cognitive load reduction}

Rosenbaum et al. (2014) conducted a study in which participants were asked to walk down an alley, pick up one of two buckets, and place the bucket on a table at the end of the alley. One of the buckets was closer to the participant than to the end table; the other bucket was closer to the end table. Rosenbaum et al. found that, regardless of which side the bucket was on (right or left side of the alley), and regardless of the weight in the buckets in the range of weights used (up to 7 pounds), participants chose to pick up the bucket closer to them on a majority of trials. In other words, participants preferred to complete the subtask of picking up a bucket earlier rather than later, even though it cost them extra physical effort to do so. This surprising result was obtained in a number of experiments, and Rosenbaum et al. called the phenomenon precrastination, introducing the term to draw a contrast with procrastination, where tasks are put off for as long as possible. In Rosenbaum et al.'s study, participants reported that they picked up the closer bucket to get the task done as soon as they could. As a result, Rosenbaum et al. speculated that participants wanted to rid themselves of the prospective memory load of picking up a bucket. Even though picking up a bucket was a cognitively trivial task, it had an associated cognitive burden, not least because of the computational demands of motor planning and perceptual-motor coordination needed to grab the bucket and carry it forward. (Lest one think of walking along, picking up a bucket, carrying it to remote site, and putting it down as cognitively trivial, recall how long it takes toddlers to learn to do this and how long roboticists have taken, and are still taking, to get robots to carry out such tasks.)

Rosenbaum et al.'s (2014) study did not explicitly involve task ordering because their participants chose between two different ways of carrying out one task - carrying a bucket to the end of an alley. Fournier et al. (2018) extended the method to the ordering of two tasks. Participants in their study were asked to carry two buckets of balls, one at a time, to an ending table to empty the balls into a bowl. The participants carried one bucket full of balls down an alley to transfer the balls to the bowl on the ending table, whereupon they brought the empty bucket back to the start position and then did the same with the remaining bucket. Like the buckets in the Rosenbaum et al. (2014) study, the two buckets were positioned at different distances from the starting position; the participants had to choose which bucket to empty first. The main result was that the bucket the participants chose first tended to be the near one rather than the far one. This preference did not depend on whether the participants could pour the balls into the bowl or had to transfer the balls one by one into the bowl. The experimenters took the latter result to mean that precrastination was driven by the desire to start the task as soon as possible rather than finish the task as quickly as possible. The authors suggested that starting a task sooner reduces cognitive effort.

In another study that involved task ordering, Fournier et al. (2019) explored the effect of cognitive load on task order choices. They asked participants to bring two objects in a line extending out from the participants' start position back to the start position. The objects were to be brought back together rather than one at a time. The participants chose between these two possible orders: (1) pick up a near object first, carry it while continuing to go forward to pick up the far object and then walk back with both objects, or (2) walk past the near object to pick up the far object first and then pick up the near object on the way back. (The choices were not explicitly enumerated for the subjects as they were in the preceding sentence.) There was a strong preference to pick up the near object first and carry it forward to the far object before bringing back both objects. This choice meant carrying the near object much farther (out and back) than if the far object were picked up first. The near-object preference occurred even when the near object weighed more than the far object.

Fournier et al. (2019) ascribed the choice to the desire to reduce cognitive load. In further support of this interpretation, they also found that the near-bucket-first preference was stronger when subjects had an extra cognitive load (holding in mind memorized digits to be recalled after returning with the objects) than when there was no such added mental load. Furthermore, the near-bucket-first preference was weaker when the objects to be carried were glasses filled with water, where it was important not to spill the water. Avoiding spillage increased the cognitive load of carrying the objects because more attention had to be paid to the glasses than when spillage was less imminent (when the glasses were emptier). Carrying the objects farther would have added greatly to the amount of attention that was required, which is presumably why participants preferred not to take the near glass first, because that would have required much more time monitoring it. Based on this reasoning and the associated result, Fournier et al. (2019) suggested that precrastination is avoided if it increases 
cognitive demands. From the memory-load experiment results, they suggested that precrastination is adopted if it reduces cognitive demands.

\section{The current study}

The results reviewed above suggest that task choices are motivated by the desire to reduce cognitive demands. However, this conclusion is based on studies that only required perceptual-motor task choices. In the current study, we explored task order choices in a situation where a cognitive task was paired with a perceptual-motor task. The main question was whether individuals would choose to complete the cognitive task earlier than (or before) the perceptual-motor task to reduce their cognitive load, as suggested by the studies reviewed above. Based on the results from Fournier et al.'s (2019) study showing that precrastination occurs more often when it reduces cognitive load, we expected participants in the current study to choose to complete a cognitively taxing task before starting a perceptual-motor (less cognitively taxing) task. These results would be consistent with the CLEAR hypothesis.

We invited our participants to choose when to perform each of $c=5,10$, or 15 category item generation tasks during $b=10$ box-moving tasks. The box-moving task was computerized. As seen in Fig. 1, boxes at the bottom of the computer screen were labeled 1 through 10 and were to be moved to one of two "tables" at the top of the screen in ascending order. One table was for odd-numbered boxes. The other was for evennumbered boxes. Participants had to move the boxes in numerical order, from 1 to 10 .

The other task was to generate a specified number of items from a given category. The number of items, $c$, was 5,10 , or 15 , and the mapping of values of $c$ to categories was balanced over participants, as detailed in the Method section. The participants were told that they could complete the categorygeneration task at a time of their choosing - before clicking on any box to be moved within the trial, after the box moving was done, or in between any of the box moves in the trial. Thus, there were 11 possible serial positions where the category generation could be done relative to the box moving on each trial. The main question was how early or late in the trial participants chose to engage in category generation. Based on the CLEAR hypothesis, we predicted that most participants would hasten to complete the item generation task and, in fact, would complete item generation before doing any boxmoving task.

By varying $c$, we could also test whether there might be a stronger tendency to complete the cognitive task earlier for larger values of $c$, as might be predicted by the CLEAR hypothesis. We also expected that as the number of to-begenerated items increased without having been done, the box-moving task would be performed more slowly. This was another CLEAR prediction.

\section{Method}

\section{Participants}

One-hundred twenty-seven undergraduate students from Illinois State University participated in exchange for course credit. Data from five participants were deleted, however: Three participants' data were deleted for failure to follow the instructions, one participant's data were deleted for failure to complete the generation task in one of the six trials, and one participant's data were deleted because of an error made by the experimenter. As a result, data from 122 participants were included in the analyses. According to $\mathrm{G}^{*}$ Power (Faul, Erdfelder, Lang, \& Buchner, 2007), a sample size of 109 participants is needed to achieve power at or above $80 \%$ with a small effect size (Cohen's $f=.10$ ).

\section{Materials, procedure, and design}

Six categories were chosen from the Van Overschelde, Rawson, and Dunlosky (2004) category norms for the generation task: four-footed animals, kitchen items, body parts, sports, clothing items, and fruits. Difficulty level of the category-generation task (i.e., number of items, $c$, to be generated from the category) was counterbalanced across participants using a Latin square design such that a similar number of participants received each of the six orders of the difficulty factor. In addition, each of the categories was assigned to each level of task difficulty $(5,10$ or 15 items) twice across the different counterbalancing programs so that different participants received a different assignment of category to task difficulty. As a result, two different categories at each level of task difficulty were presented, and in a different order across participants.

For the box-moving task, numbered cubes were positioned in a random sequence at the bottom of the computer screen on the starting table (see Fig. 1). Two boxes were shown at the top of the screen to represent tables. One was labeled "odds table" (at the top left), and the other was labeled "evens table" (top right). Boxes were shown in blue at the start of the trial, but changed to purple when the participant clicked on the correct box to be moved next (the next one in the number sequence). The numbered box then appeared in the upper table when the correct table was clicked on by the participant (see Fig. 1). A different random sequence of boxes on the starting table was shown on each trial.

After completing the consent process, participants were given instructions for the box moving task. They were told that 10 blue boxes would be shown on the starting table at the 
a

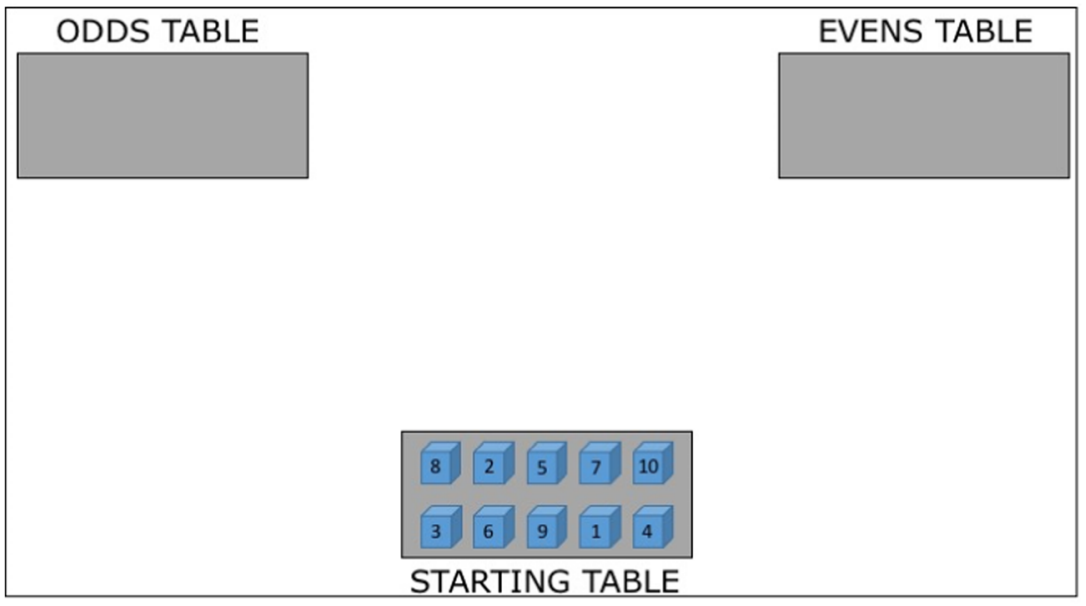

b

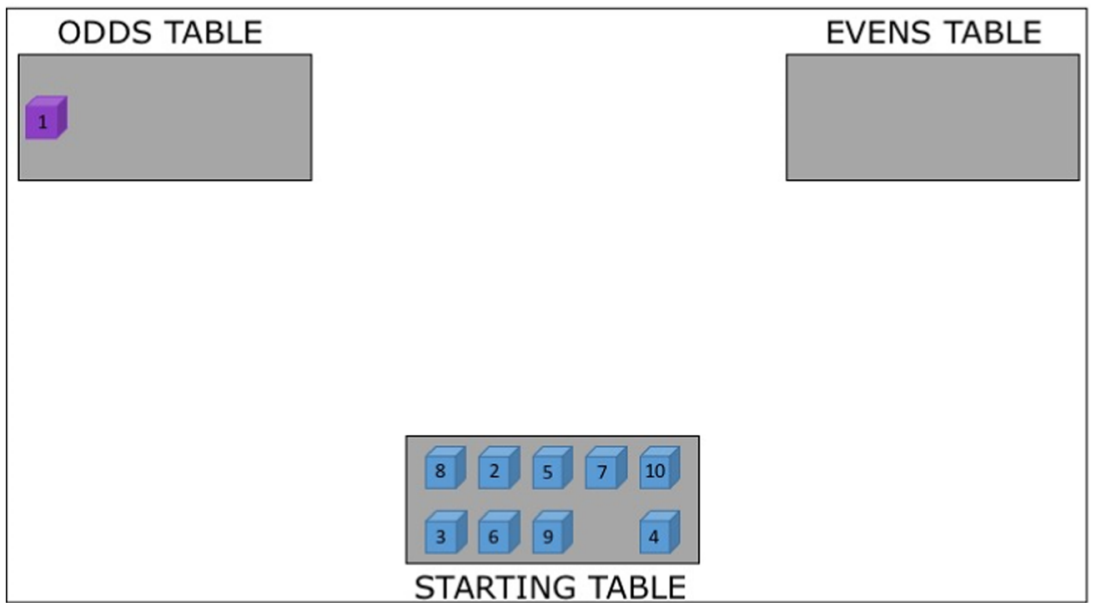

Fig. 1 The box-moving task. a Starting screen for the task with numbered boxes at the bottom of the screen. b Screen after the first box has been moved to the correct table. Participants were able to generate category items before beginning the task (a), before they clicked on the next box to be moved (b), or after any of the remaining nine boxes had been moved (not shown here) bottom of the screen, that the boxes would be in a random order, and that their task was to move the boxes in numerical order to the tables at the top of the screen according to the number on the box. Boxes labeled with odd numbers were to be moved to the odds table, and boxes labeled with even numbers were to be moved to the evens table. Participants were told to click on the correctly numbered box in ascending order, and that once they clicked on the correct box it would turn purple. As soon as the box turned purple in the bottom area, they were to move the box to the corresponding ending table by using the mouse to click on that table, whereupon the box appeared there. Participants were asked to complete the task as quickly and accurately as possible. The randomization of the numbering of the boxes differed for each trial to control for practice effects based on location. The same randomization was used for all participants so that we could examine the effects of variation in the generation task alone.
Before the generation task was introduced, participants completed a practice trial with the box-moving task. After that, when the generation task was introduced, they were told that they would be asked to generate 5,10 , or 15 examples from various categories in each of the forthcoming trials. They were told that they could say their examples out loud to the researcher whenever they chose to do so during the trial, but this would have to be before they clicked on one of the starting table boxes on the bottom table during the trial and that they would have to say all their items together before continuing the box-moving task. For example, they could say their list before clicking on any of the boxes, after moving all of the boxes, or in between, after they had moved one of the boxes to the table at the top (see Fig. 2). However, they had to generate all items at once before continuing with the box-moving task. Participants were told that they would be instructed before each trial what category would be the item source and how 


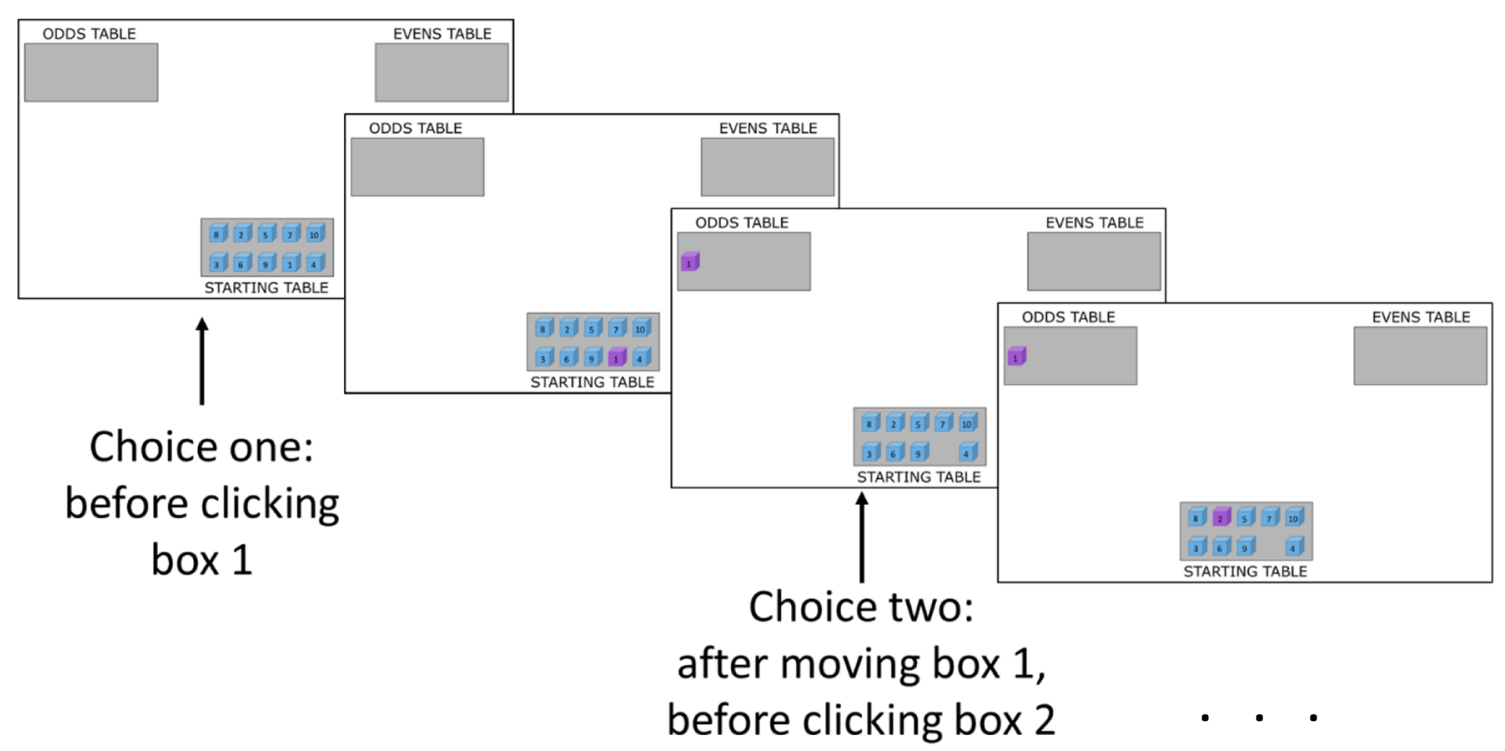

Fig. 2 Schema of the screens in the box-moving task. The figure shows the first two choice points for generating the category items. Choice points continued up to after the last (10th) box move. Category instruction (name of category and number of items to generate) was counterbalanced

many items were to be generated. They were also told to press the "g" key before they generated their category items. ${ }^{1}$

\section{Results}

\section{Manipulation check}

To check that generating more items was more difficult than generating fewer items, we conducted a repeated-measures ANOVA that examined the effect of the requested category size on percentage correct recall relative to the requested number of items. Where violations of sphericity occurred in all analyses, we report adjusted $d f$ values from the GreenhouseGeisser correction. There was a main effect of task difficulty, $F(1.109,134.142)=10.75, p<.001, \eta_{\mathrm{p}}^{2}=.08$. Pairwise comparisons revealed significant differences between $5(M=$ $100 \%, S E=0)$ and $15(M=97.32 \%, S E=.78)$ items $(p=$ $.003)$, as well as between $10(M=99.63 \%, S E=.21)$ and 15 items $(p=.005)$. Thus, as expected, the larger the number of items needed, the harder it was to generate that number of

\footnotetext{
${ }^{1}$ We had planned to use the times of the "g" presses relative to the other (mouse click) times to inquire into the temporal dynamics of our participants' thinking, but were unable to do so due to participants' inconsistent timing in pressing the " $\mathrm{g}$ " key. Instead of always pressing the key before starting to name category items as requested, they often did so in the midst of naming the items or after doing so, or they forgot to do so at all. We decided to let these mistakes go to avoid placing too much emphasis on this aspect of the task. Accordingly, we did not analyze these times. Also, the participants were not video or audio recorded as they did the task, so those potential sources of data were unavailable to us.
}

in a Latin square design with six possible condition orders that included six different categories and two trials of each difficulty level $(5,10$, and 15)

items, although the percentage correct difference for 5 and 10 items was not significant. $^{2}$

\section{Precrastination}

We next examined the selected trial position of the generation task. We defined trial position as the number of the box that was clicked on after generation of the category items: 1 if the items were generated before clicking on Box Number 1, 5 if the items were generated before clicking on Box Number 5 , and so on. A value of 11 meant that participants generated the items after moving all 10 boxes.

Figure 3 shows the mean trial positions for item generation. Because Trial Position 6 was the halfway point, we assumed this value represented neither precrastination nor procrastination. We conducted a one-sample $t$ test for each level of task difficulty to compare means with the expected value of 6 , which would indicate neither precrastination nor procrastination of the category-generation task. All three levels of task difficulty showed early generation: 5 items, $t(121)=10.12, p$ $<.001, d=.92 ; 10$ items, $t(121)=8.76, p<.001, d=.79$; and 15 items, $t(121)=8.29, p<.001, d=.75$. Figure 4 presents the frequency distributions of trial positions chosen by level of $c$ and across all levels of $c$. These results support the CLEAR hypothesis. Participants precrastinated (i.e., completed the generation task before beginning the box-moving task) on a majority of the trials in the experiment.

We next conducted a mixed-design ANOVA for trial position, with task difficulty as the within-subjects factor and order

\footnotetext{
${ }^{2}$ All pairwise comparisons included a Bonferroni correction.
} 


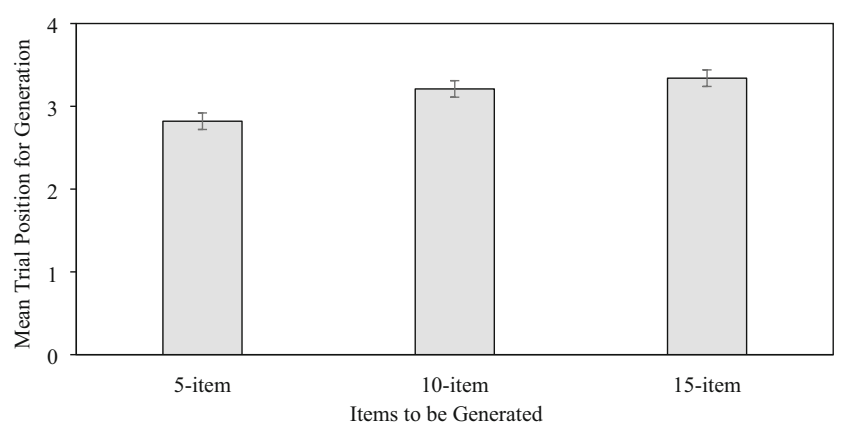

Fig. 3 Mean trial position $\pm 1 S E$ for category example generation chosen by participants when 5,10 , or 15 items were to be generated. Mean trial position for the 5 -item condition was significantly earlier than the 10 -item and 15 -item conditions

of conditions as the between-subjects factor. The ANOVA yielded a main effect of task difficulty, $F(1.899,220.304)=$ $4.72, p=.01, \eta_{\mathrm{p}}{ }^{2}=.04$. Pairwise comparisons revealed that participants completed the generation task earlier when the task was easier. Trial position was significantly lower in the 5 -item condition than in the 10-item $(p=.03)$ and 15-item $(p=$ $.02)$ conditions. However, there was no main effect of order, $F(5,116)=1.78, p=.12, \eta_{\mathrm{p}}{ }^{2}=.07$, and no interaction between the factors, $F(9.496,220.304)<1.0, p=.86, \eta_{\mathrm{p}}{ }^{2}=.02$. Thus, the order in which the participants completed the conditions did not affect the propensity for early generation. These results are not consistent with the CLEAR hypothesis, which predicted earlier generation as task difficulty increased. We obtained the opposite trend. It is possible that this result is

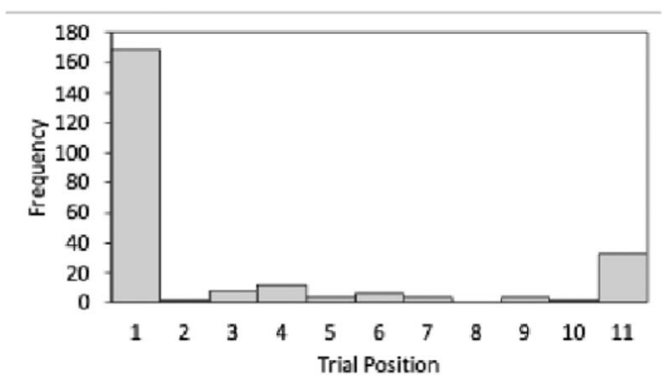

$\mathbf{C}-c=15$

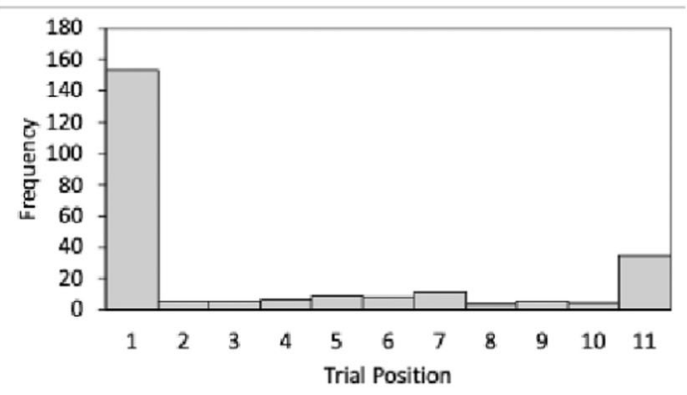

an artifact of the cognitive task used in the current experiment. Participants may have delayed generation until they had mentally simulated a number of items in the 10-item and 15-item conditions. We consider this possibility in the analysis of response times below.

\section{Response times}

The next analysis concerned the times for the box transfers. These were the times for clicking the correct box at the bottom of the screen, and then clicking on the correct ending table at the top of the screen. Boxes that were moved immediately after category-item generation were not included in the time calculations. The mean times for each item difficulty condition were calculated and are shown in Table 1 . We conducted a mixed-factor ANOVA for the times, with the factors number of items needed and order of conditions. The results showed that the times were not significantly affected by item difficulty, $F(1.118,129.688)=2.12, p=.16, \eta_{\mathrm{p}}{ }^{2}=.02$, or order, $F(5,116)<1.0, p=.57, \eta_{\mathrm{p}}{ }^{2}=.03$, and these factors did not interact, $F(5.59,129.688)<1.0, p=.47, \eta_{\mathrm{p}}{ }^{2}=.04$. These results also suggest that it is unlikely participants who generated items after starting the box-moving task were generating items subvocally while moving boxes because this should have caused them to move boxes slower as task difficulty increased due to interference.
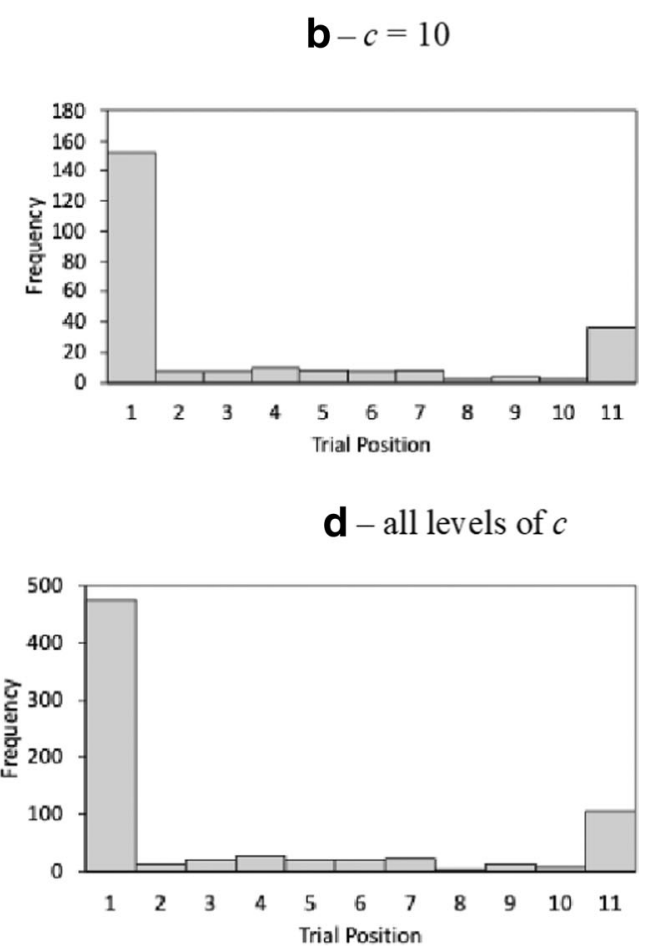

Fig. 4 Frequency of the different trial positions chosen by participants for the generation task across all trials. a-c Frequency distribution by task difficulty condition. d Overall frequency distribution for all levels of $c$ combined 
Table 1 Mean box movement completion times (ms) by generation task difficulty and participants' consistency group with standard errors in parentheses

\begin{tabular}{llllll}
\hline & Consistency group & & & \\
\cline { 2 - 5 } & $\begin{array}{l}\text { Inconsistent } \\
n=44\end{array}$ & $\begin{array}{l}\text { Consistent-beginning } \\
n=61\end{array}$ & $\begin{array}{l}\text { Consistent-middle } \\
n=3\end{array}$ & $\begin{array}{l}\text { Consistent-end } \\
n=14\end{array}$ \\
\hline 5 item & $2,523(62)$ & $2,432(53)$ & $2,544(134)$ & $2,803(110)$ & $2,941(111)$ \\
10 item & $2,651(56)$ & $2,444(53)$ & $2,640(250)$ & $2,933(383)$ & $2,581(40)$ \\
15 item & $2,984(216)$ & $2,468(183)$ & $2,949(62)$ & $2,719(129)$ \\
\hline
\end{tabular}

\section{Group classification}

Besides showing the overall times for the box-moving task, Table 1 also reflects a classification of participants in terms of how consistent they were in when they chose to generate items. Participants were assigned to one of four groups based on their choice: consistent beginning (they generated their category items before starting the box-moving task on all trials, $n=61$ ); consistent end (they generated their category items after moving all 10 boxes on all trials, $n=14$ ); consistent middle (they generated their category items before the same box number between 1 and 10 on every trial, $n=3$ ); or inconsistent (they generated their category items at different positions across the trials, $n=44$ ). The consistent-middle group was too small to include in the analyses, but the other three groups were large enough to include them as a between-subjects factor in a mixed ANOVA that evaluated the relation between task difficulty and group. There was no main effect of task difficulty, $F(1.114,129.195)=$ $1.30, p=.26, \eta_{\mathrm{p}}^{2}=.01$, or interaction between the factors, $F(2.272,129.195)<1.0, p=.39, \eta_{\mathrm{p}}^{2}=.02$, but times differed significantly across groups, $F(2,116)=4.75, p=$ $.01, \eta_{\mathrm{p}}^{2}=.08$. Pairwise comparisons showed that participants in the consistent-beginning group $(M=2,448 ; S E=$ 75) completed the box task more quickly than did participants in the consistent-end group $(M=2,893 ; S E=156$; ( $p=.04)$, and marginally more quickly than participants in the Inconsistent group $(M=2,720 ; S E=88 ; p=.06)$. Table 1 shows the mean response times by group and level of task difficulty.

\section{Correlations}

Whereas the foregoing analysis concerned the effect of group and task difficulty on performance times, the final analysis concerned the relations between the trial positions that participants chose for their item generations and times for the box transfers for each level of task difficulty. As generation was completed later in the trial, time taken to move the boxes increased. Significant positive relationships were found for all levels of task difficulty: 5 items: $r(120)=+.27, p=.002 ; 10$ items: $r(120)=+.40, p<.001$; and 15 items, $r(120)=+.25, p=.006 .^{3}$

\section{Discussion}

In the present study, we investigated the sequencing of cognitive tasks and perceptual-motor tasks. We asked our participants to choose when to generate $c=5,10$, or 15 items from various semantic categories relative to a task involving $b=10$ successive box transfers from the bottom of a computer screen to the top of the screen. We found that exactly half of our participants (61 of 122) always completed the generation task before starting the box-moving task. Only 17 participants consistently completed the generation task in the middle or end of the box-moving trials. On average, participants completed the cognitive task before or during the first half of the movement trials, and this was true in all task difficulty conditions. The results are strikingly consistent with the main prediction of the CLEAR hypothesis.

Also consistent with the CLEAR hypothesis was the finding that there was a speed cost to the perceptual-motor task when participants completed the cognitive task later in the trial. The participants in the consistent-end and inconsistent groups performed the box-moving task more slowly than the consistent-beginning group did (see Table 1), in accord with the view that having multiple tasks on one's mind interferes with performance.

One result was not consistent with the CLEAR hypothesis. According to the CLEAR hypothesis, participants would perform the cognitive task earlier with increasing values of $c$. This prediction was not supported. Instead, participants completed the cognitive task later for higher values of $c(c=5 \mathrm{vs}$. $c$ $=15$ ), perhaps because participants found the $c=15$ condition particularly taxing and chose to reduce their overall cognitive load by searching their memory for category items while they began moving boxes. This could have resulted in slower boxmoving times, but no difference in response times was evident

\footnotetext{
${ }_{3}^{3}$ Because many of the participants consistently generated category items before beginning or after completing the box-moving task, there were not enough participants with times both before and after category generation to compare pregeneration and postgeneration times.
} 
in the current study across levels of task difficulty. Future research will address the participants' reasoning in their task order choices. However, this effect of task difficulty does allow us to rule out the suggestion that the category-generation task was performed earlier because it was more rewarding than the box-moving task. If the generation task was inherently more rewarding, we would have expected consistently early performance of this task.

Notwithstanding this somewhat problematic result, our findings generally support the core conclusion of Fournier et al. (2019), Fournier et al. (2018), and Rosenbaum et al. (2014) that precrastination reduces cognitive load. Our study also contributes to a growing body of data showing interactions between higher order cognitive and perceptual-motor tasks. One such study (Zhang, Wininger, \& Rosenbaum, 2014) showed task interference across concurrent cognitive tasks (very similar to the task used in the current study) and a perceptual-motor task (moving an object back and forth with one hand). Although the motor task did not affect performance in the cognitive task (relative to the cognitive task performed alone), generating words while performing the motor task slowed hand movements relative to the motor task performed alone. The results of Zhang et al. (2014) are similar to our results in showing slowed box-moving speed for participants who held on to the intention to complete the item-generation task until the end of the box-moving trial (i.e., the consistentend group). Although generation was not simultaneous with the perceptual-motor task in our study, the results of both studies show interference of a cognitive task on movements in a perceptual-motor task. The fact that a majority of our participants chose to complete the generation task before the box-moving task might have reflected a desire to reduce this interference.

Our results also fit with another study showing that perceptual-motor performance has its own significant memory demands. Weigelt, Rosenbaum, Huelshorst, and Schack (2009) investigated end-state comfort and hysteresis in motor planning (see Rosenbaum \& Feghhi, 2019) and reported an interesting effect on a common memory phenomenon - the serial position curve (Glanzer \& Cunitz 1966). Participants in the study were asked to pick up cups from drawers and memorize the letters inside the cups. The cups were positioned upside down in the drawers, causing the participants to invert each cup after picking it up to look at the letter inside and then replace it in the drawer in its original inverted position. The results showed no effect of memorization on end-state comfort or hysteresis, but the recency effect - the tendency to recall items from the end of a memorized list better than items in the middle of the list - was eliminated in the participants' memory performance. This study shows, like the Zhang et al. (2009) study did, that perceptual-motor tasks and tasks that are more classically considered "cognitive" (memorization and item generation) share cognitive resources. It makes sense, then, that people prefer to "clear their minds" before doing perceptual-motor tasks, such as moving boxes on a computer display.

Our results fit with the notion that people prefer less demanding mental tasks over more demanding ones (Kool et al., 2010; Potts, Pastel, \& Rosenbaum, 2018). This preference has been shown in studies employing other cognitive tasks such as the delay-execute procedure (Einstein et al., 2000), where participants are asked to delay their prospective memory responses for a short time after presentation of a prospective memory cue. In one study that used this procedure, McDaniel et al. (2003) found that a number of participants failed to delay their prospective memory responses but instead responded as soon as the prospective memory cue was presented. Violating the instruction in this way nonetheless relieved the cognitive load of the task.

Consistent with the idea that precrastination allows reduction of cognitive load, differences in working memory abilities could influence individuals' tendency to precrastinate. The current finding of consistent-beginning and consistent-end groups of participants, who performed the box-moving task with different speeds, suggests that individual differences may relate to task order choices. This is an interesting avenue for future work in this area.

Another study by Potts et al. (2018) also showed a preference to reduce cognitive load. These researchers found that when given a choice between a perceptual-motor task and a cognitive task (carrying a bucket vs. counting), participants chose the motor task more often as the difficulty of the cognitive task increased (the higher the count). These findings are consistent with the CLEAR hypothesis and highlight the preference for reduction of cognitive load. The results we have presented indicate that this preference has profound implications for task ordering.

Author note Supported in part by a University of California, Riverside, Committee on Research grant to the last author.

\section{References}

Blinch, J., \& DeWinne, C. R. (2019). Pre-crastination and procrastination effects occur in a reach-to-grasp task. Experimental Brain Research, 237(5), 1129-1139. https://doi.org/10.1007/s00221-019-05493-3

Einstein, G. O., \& McDaniel, M. A. (2005). Prospective memory: Multiple retrieval processes. Current Directions in Psychological Science, 14, 286-290.

Einstein, G. O., McDaniel, M. A., Manzi, M., Cochran, B., \& Baker, M. (2000). Prospective memory and aging: Forgetting intentions over short delays. Psychology and Aging, 15, 671-683.

Faul, F., Erdfelder, E., Lang, A.-G., \& Buchner, A. (2007). G*Power 3: A flexible statistical power analysis program for the social, behavioral, and biomedical sciences. Behavior Research Methods, 39, 175-191.

Fournier, L. R., Coder, E., Kogan, C., Raghunath, N., Taddese, E., \& Rosenbaum, D. A. (2019). Which task will we choose first? Precrastination and cognitive load in task ordering. Attention, 
Perception, \& Psychophysics, 81(2), 489-503. https://doi.org/10. 3758/s13414-018-1633-5

Fournier, L. R., Stubblefield, A. M., Dyre, B. P., \& Rosenbaum, D. A. (2018). Starting or finishing sooner? Sequencing preferences in object transfer tasks. Psychological Research Psychologische Forschung https://doi.org/10.1007/s00426-018-1022-7

Glanzer M \& Cunitz AR (1966) Two storage mechanisms in free recall. Journal of Verbal Learning and Verbal Behavior, 5, 351-360.

Kool, W., \& Botvinick, M. M. (2014). A labor/leisure tradeoff in cognitive control. Journal of Experimental Psychology: General, 143, $131-141$.

Kool, W., McGuire, J. T., Rosen, Z. B., \& Botvinick, M. M. (2010). Decision making and the avoidance of cognitive demand. Journal of Experimental Psychology: General, 139, 665- 682.

McDaniel, M. A., Einstein, G. O., Stout, A. C., \& Morgan, Z. (2003). Aging and maintaining intentions over delays: Do it or lose it. Psychology and Aging, 18, 823-835.

Potts, C. A. Pastel, S., \& Rosenbaum, D. A. (2018). How are cognitive and physical difficulty compared? Attention, Perception, \& Psychophysics, 80, 500-511.

Rosenbaum, D. A., \& Feghhi, I. (2019). The time for action is at hand. Attention, Perception, \& Psychophysics https://doi.org/10.3758/ s13414-018-01647-7

Rosenbaum, D. A., Fournier, L. R., Levy-Tzedek, S., McBride, D. M., Rosenthal, R., Sauerberger, K., . . . Zentall, T. R. (2019). Sooner rather than later: Precrastination rather than procrastination. Current Directions in Psychological Science. https://doi.org/10.1177/ 0963721419822652
Rosenbaum, D. A., Gong, L., \& Potts, C. A. (2014). Pre-crastination: Hastening of subglobal completion at the expense of extra physical effort. Psychological Science, 25, 1487-1496. https://doi.org/10. 1177/0956797614532657

Van Overschelde, J. P., Rawson, K. A., \& Dunlosky, J. (2004). Category norms: An updated and expanded version of the Battig and Montague (1969) norms. Journal of Memory and Language, 50, 289-335.

Weigelt, W., Rosenbaum, D. A., Huelshorst, S., \& Schack, T. (2009). Moving and memorizing: Motor planning modulates the recency effect in serial and free recall. Acta Psychologica, 132, 68-79.

Zhang, L., Wininger, M., \& Rosenbaum, D. A. (2014). Word generation affects continuous hand movements. Journal of Motor Behavior, 46, $115-123$.

Open practices statementThe experiment was not preregistered; data and materials are available from the corresponding author.

Publisher's note Springer Nature remains neutral with regard to jurisdictional claims in published maps and institutional affiliations. 